\title{
Effects of weight and definiteness on speakers' choice of clausal ordering in English ${ }^{1}$
}

\author{
Elaine J. Francis \\ Purdue University \\ ejfranci@purdue.edu
}

\author{
Laura A. Michaelis \\ University of Colorado at Boulder \\ Laura.Michaelis@colorado.edu
}

\section{Introduction}

In Relative Clause Extraposition (henceforth RCE), a subject-modifying relative clause occurs following the VP rather than adjacent to the noun it modifies, as in this example from the ICE-GB corpus:

Further research has been conducted on this that indicates this criticism may not be just.

Why should English speakers sometimes prefer a discontinuous structure as in (1) when an adjacent ordering could express the same meaning? Most previous research has focused on discourse-based explanation: RCE is preferred when the subject NP is focal and/or the VP is backgrounded, accounting for the tendency of RCE tokens to contain passive or unaccusative predicates (Kuno \& Takami 2004; Rochemont \& Culicover 1990) and indefinite subjects (Huck \& Na 1990). However, a more recent study by Francis (2010) offered an alternative explanation: corpus and reading-time data showed an advantage for RCE when the VP was short relative to the RC, fitting the general observation that late placement of 'heavy' constituents facilitates production and comprehension (Hawkins 2004; Wasow 2002).

While previous studies have investigated discourse and weight-based factors in isolation, the current study examined the combined effects of definiteness (a major correlate of discourse status) and constituent length on speakers' choice of clausal ordering. Three experiments are reported here: a structural preference task, and two elicited production tasks.

\section{Experiments 1-2}

\section{Methods}

The first two experiments, which used the same sentence materials in different tasks, manipulated three factors in a repeated measures design: (1) definiteness (the vs. some), (2) RC length (5 words vs. 12 words), and (3) VP length ( 2 words vs. 5 words). All combinations of these factors resulted in eight experimental conditions, which were repeated across eight lexicalizations (token sets), for a total of 64 experimental items. The following passive verbs were used as the main verb for each set: conducted, raised, formed, provided, considered, presented, received, and made. Filler sentences (96 in Experiment 1, and 64 in Experiment 2) were used to distract participants from the structure being tested.

Sample sentences for Conditions 1 and 8 are illustrated below in (2) and (3). Participants were expected to prefer RCE most often in Condition 1, and least often in Condition 8, with the other six conditions being between these two extremes. That is, in (2), sentence (a) should be preferred most often, while in (3), sentence (b) should be preferred most often.

\footnotetext{
${ }^{1}$ We are grateful to our research assistants, Corinne Feight, Xuebo Gong, Charles Lam, and Sunny Park, and we thank Jyotishka Datta for consulting with us on the statistics. We also gratefully acknowledge the Department of English and the Linguistics Program at Purdue University and the Department of Linguistics at the University of Colorado for financial support of this project.
} 
(2) Condition 1: indefinite subject NP, short VP, long RC

a. Some research was conducted that refutes the existing theories with very clear and convincing new evidence.

b. Some research that refutes the existing theories with very clear and convincing new evidence was conducted.

(3) Condition 8: definite subject NP, long VP, short RC

a. The research has been conducted fairly recently that refutes the existing theories.

b. The research that refutes the existing theories has been conducted fairly recently.

Experiment 1: Thirty native speakers of American English, ages 18-53 (mean age 22) were recruited from the Purdue University community to participate in Experiment 1. Each was paid $\$ 8$ for a session of about 35 minutes. This experiment measured structural preference in reading, following a procedure similar to the one used in Rosenbach (2005). Participants were presented with a written questionnaire that contained all of the experimental and filler sentences. For each item on the questionnaire, they were asked to choose which of two versions a sentence sounded more natural (as in $2 a-b$ and $3 a-b$ above). Items were arranged in blocks to avoid similar sentences occurring together, and the order of items within each block was randomized. Eight versions of the questionnaire were created with different orderings of blocks. Top-bottom ordering of (a-b) options (as in 3a-b above) was counterbalanced across items and across participants.

Experiment 2: Thirty-one native speakers of American English, ages 18-57 (mean age 25) were recruited from the Purdue University community to participate in Experiment 2. Each was paid $\$ 10$ for a session of about 45 minutes. Experiment 2 measured structural preference in production, following Stallings et al (1998). Participants saw sentence constituents arranged on the computer screen from top to bottom (for example, VP, NP, RC) and were asked to formulate and speak a sentence in one of two orders, depending on their personal preference: middle-top-bottom, or middle-bottom-top. Similar to Experiment 1, items were arranged in blocks. The order of items within each block and the order of blocks were randomized separately for each participant by the E-Prime experiment program. The topbottom ordering of the constituents (i.e. VP top, RC bottom or vice versa) was counterbalanced across items and participants.

\section{Results}

Experiment 1: As shown in Figure 1, RCE was preferred most often (74\%) with short VP, long RC, and indefinite subject, and least often with a long VP, short RC, and definite subject (31\%), as expected. Using a binary logistic regression analysis, significant main effects were found for RC length $\mathrm{X}^{2}(1)=$ 32.12, $\mathrm{p}<0.01$, and definiteness $\mathrm{X}^{2}(1)=120.25, \mathrm{p}<0.01$, but there was no significant effect of VP length. Top-bottom ordering of the (a-b) options was also not significant, and there were no significant interactions.

Experiment 2: As shown in Figure 2, the results for Experiment 2 were similar but not identical to the results of Experiment 1. RCE was preferred most often (55\%) with short VP, long RC, and indefinite subject, and least often with a long VP, short RC, and definite subject (13\%). There was again a significant main effect of definiteness, $\mathrm{X}^{2}(1)=62.42, \mathrm{p}<0.01$, but length effects were reversed: VP length was a significant factor, $\mathrm{X}^{2}(1)=7.21, \mathrm{p}<0.01$, while RC length was not. Similar to Experiment 1, top-bottom ordering of the constituents was not significant, and there were no significant interactions. 


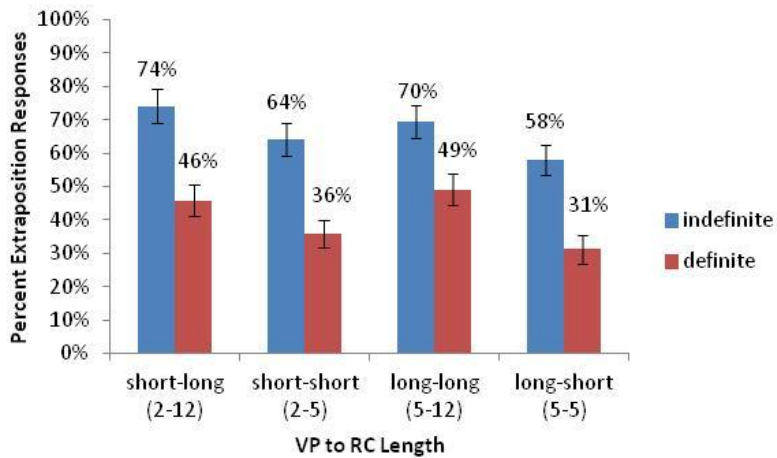

Figure 1: Percentage of RCE responses in a preference task

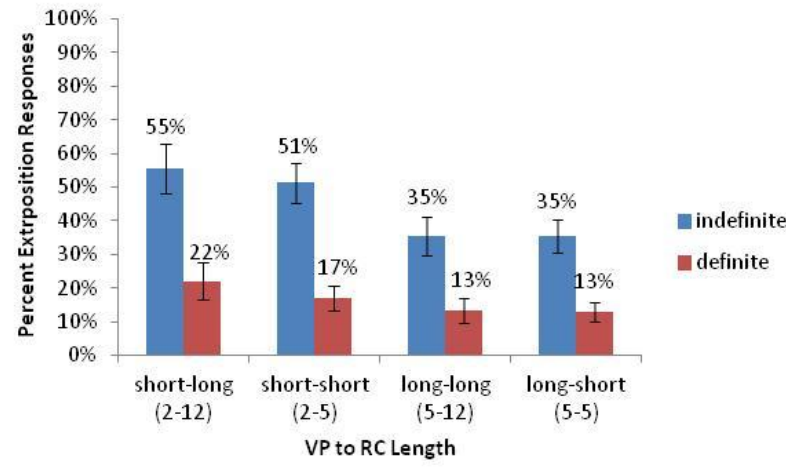

Figure 2: Percentage of RCE responses in a production task (no memorization)

\section{Experiment 3}

The results of Experiment 2 were puzzling in that RC length was found not to be significant. We thought that participants might have ignored RC length in making their decisions about word order because they were not required to memorize the phrases. Rather, since the phrases were re-displayed on the screen during the speaking phase, participants might simply have been reading the long RCs aloud. Experiment 3 was designed as a follow-up to Experiment 2 to test whether RC length would become a significant factor when participants were required to memorize the phrases before producing the sentence.

\section{Methods}

Thirty-five native speakers of American English, ages 18-33 (mean age 21) were recruited from the Purdue University community to participate in Experiment 3. Each was paid $\$ 5$ for a session of about 20 minutes. Experiment 3 was similar to Experiment 2, except that only VP length (2 words vs. 5 words) and RC length (4 words vs. 10 words) were manipulated. All test sentences had an indefinite subject and a passive main verb. A modified version of the production task from Experiment 2 was used: participants were required to memorize the constituents and speak the sentence without any visual cues. The ordering and presentation of the stimuli was similar to Experiment 2.

\section{Results}

As shown in Figure 3, preliminary results from 22 participants show that VP length was again a significant factor influencing speakers' choice of structure, $\mathrm{X}^{2}(1)=16.54, \mathrm{p}<0.01$. Participants preferred RCE more often with short VPs than with long VPs. Although the effect was not as strong as in Experiment 1, there was also a main effect of RC length, $X^{2}(1)=4.78$, $\mathrm{p}=0.03$, thus confirming that the lack of effect in Experiment 2 was likely due to the task. Similar to Experiments 1-2, top-bottom ordering of the constituents was not significant, and there were no significant interactions. 


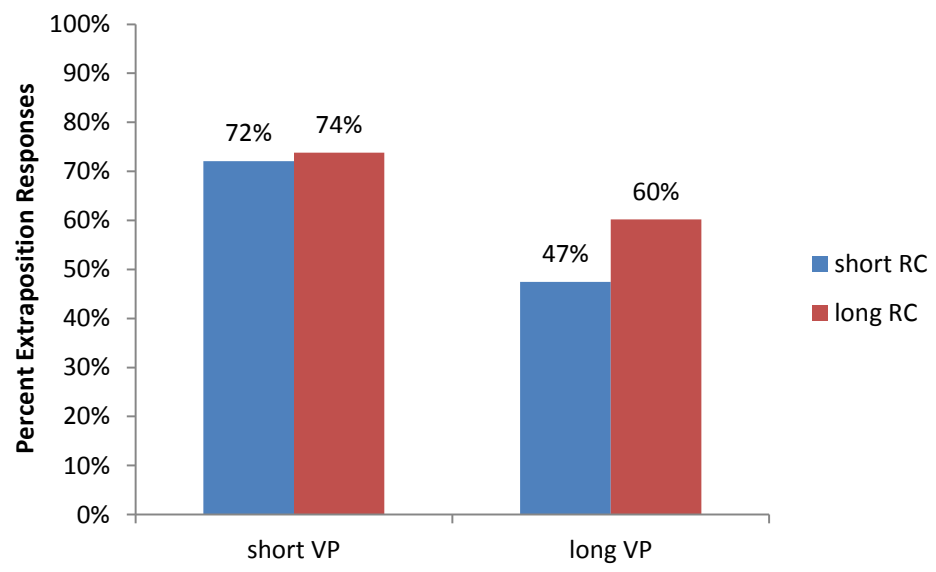

Figure 3: Percentage of RCE responses in a production task (with memorization)

\section{Discussion}

These experiments established independent effects of constituent length and definiteness on speakers' choice of RCE vs. non-RCE structure in language use. The effect of definiteness was consistently strong, while the exact length effect differed depending on the task. A stronger effect of RC length was found for the written questionnaire, consistent with the RC length effect found in an earlier acceptability judgment task (Francis 2010), while a stronger effect of VP length was found for the elicited production task, consistent with the stronger VP length effect in an earlier corpus study (Francis 2010). Thus, we conjecture that RC length may be more important in comprehension of these sentences, while VP length may be more important in production. Discourse-related factors such as definiteness appear to be equally important for comprehension and production. Further research is needed to verify these preliminary findings.

\section{References}

Francis, Elaine J. 2010. Grammatical weight and relative clause extraposition in English. Cognitive Linguistics 21: 35-74.

Hawkins, John A. 2004. Efficiency and Complexity in Grammars. Oxford \& New York: Oxford University Press.

Huck, Geoffrey J. \& Younghee Na. 1990. Extraposition and focus. Language 66(1): 51-77.

Kuno, Susumu \& Ken-ichi Takami. 2004. Functional Constraints in Grammar: On the Unergative-unaccusative Distinction. Amsterdam \& Philadephia: John Benjamins.

Rochemont, Michael S. \& Peter W. Culicover. 1990. English Focus Constructions and the Theory of Grammar. Cambridge: Cambridge University Press.

Rosenbach, Anette. 2005. Animacy versus weight as determinants of grammatical variation in English. Language 81(3): 613-644.

Stallings, Lynne M., Maryellen C. MacDonald \& Pedraig G. O’Seaghdha. 1998. Phrasal ordering constraints in sentence production: Phrase length and verb disposition in heavyNP shift. Journal of Memory and Language 39(3): 392-417.

Wasow, Thomas. 2002. Postverbal Behavior. Stanford: CSLI Publications. 